\title{
Adhesion Interaction between Atomically Defined Tip and Sample
}

\author{
G. Cross, A. Schirmeisen, A. Stalder, and P. Grütter* \\ Center for the Physics of Materials, Department of Physics, McGill University, Montréal, Canada
}

\author{
M. Tschudy and U. Dürig* \\ IBM Research Division, Zurich Research Laboratory, CH-8803 Rüschlikon, Switzerland
}

(Received 24 November 1997)

\begin{abstract}
We have measured forces between an atomically defined W(111) tip and an $\mathrm{Au}(111)$ sample in ultrahigh vacuum at $150 \mathrm{~K}$. The $\mathrm{W}$ tips are manipulated and characterized on an atomic scale both before and after sample approach by field ion microscopy. Forces between the tip and the sample are measured by an in situ differential interferometer. We observe strong attractive adhesion forces, which turn repulsive upon the further approach of the tip towards the Au surface. Unexpected for a metallic system, there is no spontaneous jump to contact. The force versus tip-sample distance curve shows only modest hysteresis, and the field ion microscopy images reveal an atomically unchanged tip apex. [S0031-9007(98)06188-2]
\end{abstract}

PACS numbers: 61.16.-d, 62.20.Dc, 68.35.Gy, 85.40.Ux

Many physical phenomena are believed to be governed by the effects of metallic adhesion. The study of those interactions can shed light on the fields of powder metallurgy, machining, and tribology, and will advance our understanding of molecular-scale lubrication. Furthermore, the study of force interactions between a tip and a sample plays a key role in understanding imaging mechanisms in scanning tunneling microscopy [1] and atomic force microscopy [2]. Moreover, tip-sample forces constitute the basic mechanism for atomic [3] and molecular-scale manipulation [4]. Tip-sample adhesion has been investigated previously [5-7]. Although the geometrical shape of the tip as well as the chemical composition of the apex surface $[6,8]$ have a significant effect on the strength and character of the interaction, in all previous investigations the structure of the tip was in fact unknown. Hence, tip fabrication and characterization on the atomic level in combination with local probe microscopies are a prerequisite for attaining full control over imaging and manipulation processes. We have taken a first step in this direction by investigating the adhesion interaction between an atomically characterized W(111) trimer tip and a $\mathrm{Au}(111)$ surface.

Experiments were conducted under ultrahigh vacuum (UHV) conditions ( $p<2 \times 10^{-11}$ mbar) and at a temperature of $150 \mathrm{~K}$. The apparatus [9] (see Fig. 1) is a scanning tunneling microscope (STM) complemented by a channel-plate-phosphorus-screen assembly mounted approximately $10 \mathrm{~cm}$ below the STM tip for in situ field ion projection imaging. The setup also includes a removable sample stage with a differential optical interferometer for sensing deflections induced in a cantilever spring, which in turn acts as a sensor for measuring tip-sample interaction forces with subnanonewton resolution. The STM and sample stage are mounted inside a copper heat shield attached to a spring bellows suspension, which acts as a combined cooling and vibration isolation stage [10].
Thin $(\approx 200 \mathrm{~nm})$ (111)-oriented Au films evaporated on mica substrates served as samples. Samples were glued onto the free end of the cantilever spring (approximately $5 \mathrm{~mm}$ long and $1 \mathrm{~mm}$ wide), which was cut from a thin glass slide (150 $\mu \mathrm{m}$ thick). The effective spring constant at the tip position was $116 \pm 10 \mathrm{~N} / \mathrm{m}$ and the resonance frequency was of the order of $500 \mathrm{~Hz}$. The tip position was determined in situ by video microscopy, and the relevant cantilever spring constant was carefully calibrated ex situ. Samples were repeatedly sputter cleaned for 3 min using $1-\mathrm{keV} \mathrm{Ne}$ ions and annealed in UHV at a temperature of $\approx 250{ }^{\circ} \mathrm{C}$. As a result, a surface topography consisting of monoatomic flat terraces a few tens of a nanometer wide was obtained. The tip was fabricated from a 0.1mm-thick (111)-oriented $\mathrm{W}$ single-crystal wire by means

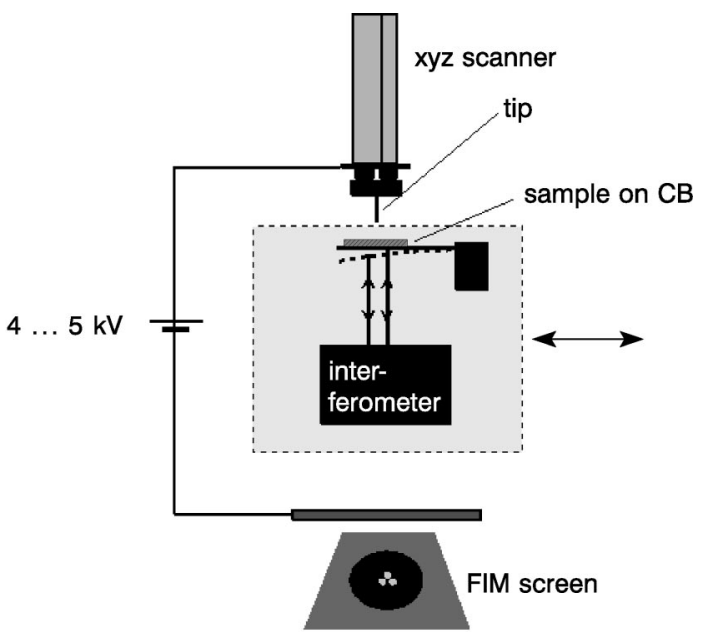

FIG. 1. Sketch of the experimental setup. The sample stage of the STM comprises a differential optical interferometer for sensing the deflection of a cantilever spring (CB), which holds the sample. The sample stage can be removed for in situ field ion projection imaging (FIM) of the tip. 
of electrochemical etching in a concentrated potassium hydroxide solution. Tip shaping involved a series of steps performed in situ. First, the tip was flash heated to approximately $1000{ }^{\circ} \mathrm{C}$ in order to remove oxides and surface contaminants. Then the tip was operated in the field emission mode for some 2 min at currents up to $5 \mu \mathrm{A}$. The apex was tailored to its final trimer shape by means of field evaporation under visual control using field ion microscopy (FIM) [11]. Typical imaging and evaporation potentials were 4.2 and $4.5 \mathrm{kV}$, respectively, and ultrapure $\mathrm{He}$ was used as imaging gas. Using these trimer tips, we obtained a remarkably stable tunneling current. In particular, noise spikes frequently associated with sharp tips were entirely absent. Furthermore, imaging of the $\mathrm{Au}(111) 22 \times \sqrt{3}$ reconstruction was readily achieved, which proves that the tip-sample distance could be reliably controlled on a $0.01-\mathrm{nm}$ level.

A typical experimental run took about $2.5 \mathrm{~h}$. This comprised the fabrication and characterization of the initial trimer tip, measurement of adhesion force curves as a function of tip-sample distance (henceforth referred to as the force curve) on a freshly prepared Au sample, and final characterization of the tip shape to check for possible irreversible tip modifications. An FIM image of the initial trimer tip used in the experiment reported here and the corresponding sphere model are shown in Figs. 2(a) and 2(c), respectively. We found that this tip structure is stable for hours; specifically no evidence of surface migration was observed. However, one rest gas atom per hour is typically adsorbed and imaged on the tip. After tip characterization, the sample stage was moved in and the tip was approached towards the sample until tunneling contact was established.

Force curves were measured on a selected flat area of the sample. Tunneling corresponding to a gap resistance of $100 \mathrm{M} \Omega$ (bias voltage $=100 \mathrm{mV}$, current $=1 \mathrm{nA}$ ) was used to define a reference position for the tip-sample separation $s$. An approach-retraction cycle started with the tip being retracted by $1.2 \mathrm{~nm}$ from the reference position. Then the tip was ramped $2.2 \mathrm{~nm}$ toward the sample and subsequently retracted by the same distance at a rate of $1.1 \mathrm{~nm} / \mathrm{s}$. Data samples of the tip-sample force were taken every $10 \mathrm{~ms}$. At the end of one such cycle, the initial tunneling mode was reestablished before the next measurement was started. Experimentally, the tip excursion with respect to the reference position $\Delta z$ is the control parameter. The actual motion of the tip relative to the sample $\Delta s$ is obtained by subtracting the lever response at the tip from $\Delta z$.

A force curve representing averaged data from 32 approach-retraction cycles is shown in Fig. 3. Its characteristic features are as follows. (1) The range of tip-sample distances over which substantial attractive forces are observed extends over more than $1 \mathrm{~nm}$, (2) hysteresis corresponding to an energy dissipation of $\approx 7 \mathrm{eV}$ (calculated from the area enclosed by the approach and retraction force curves) is observed in the range of

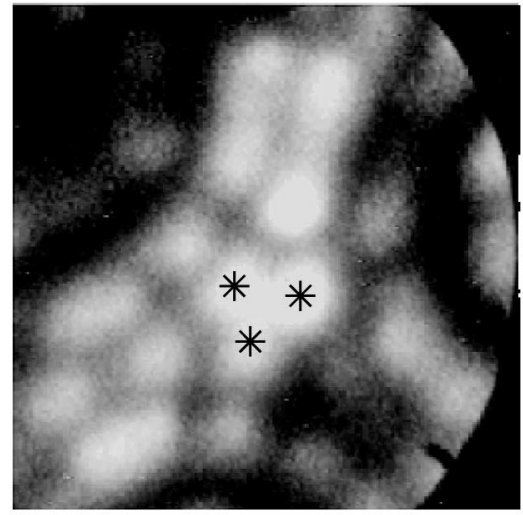

(a)

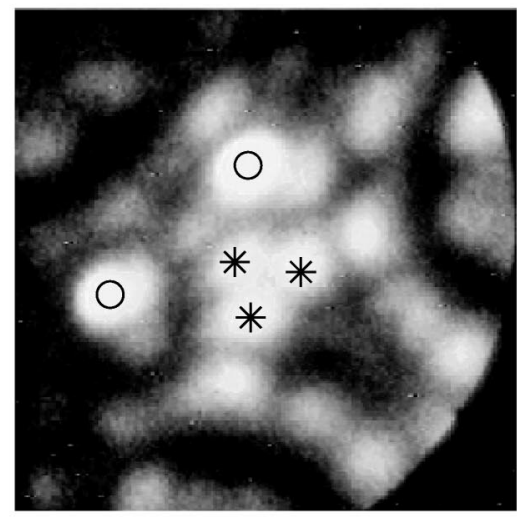

(b)

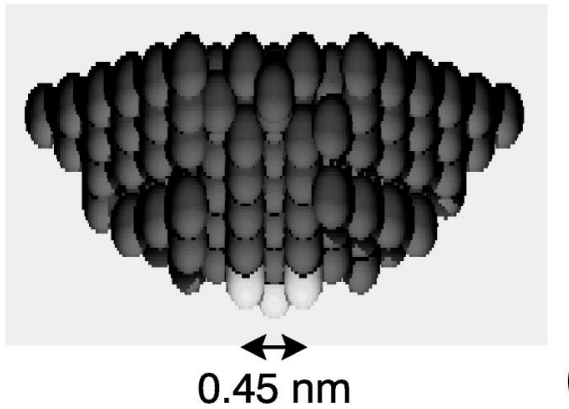

(c)

FIG. 2. Field ion projection image of the (111)-oriented W tip (a) before and (b) after the adhesion experiment. Trimer atoms are marked with an asterisk (*). Note that with the exception of two additional adatoms [bright spots marked with a circle (o)] no irreversible tip changes occurred even though the apex atoms had been exposed to tensile and compressive forces of the order of $2 \mathrm{nN}$ per atom, corresponding to uniaxial contact stresses of the order of $25 \mathrm{GPa}$. The two atoms marked with $\circ$ are compatible with rest gas adsorbed during the 2.5-h lapse between acquiring image (a) and (b). The variations in intensities between (a) and (b) are due to the strong dependence of FIM image intensities on the local atomic structure such as that introduced by the two adsorbates. (c) Hard-sphere model of the tip apex reconstructed from FIM images (apex trimer highlighted in bright tones, vertical scale is expanded by a factor of 1.8).

maximum adhesion interaction, (3) there is no evidence of a spontaneous jump to contact, which normally occurs 


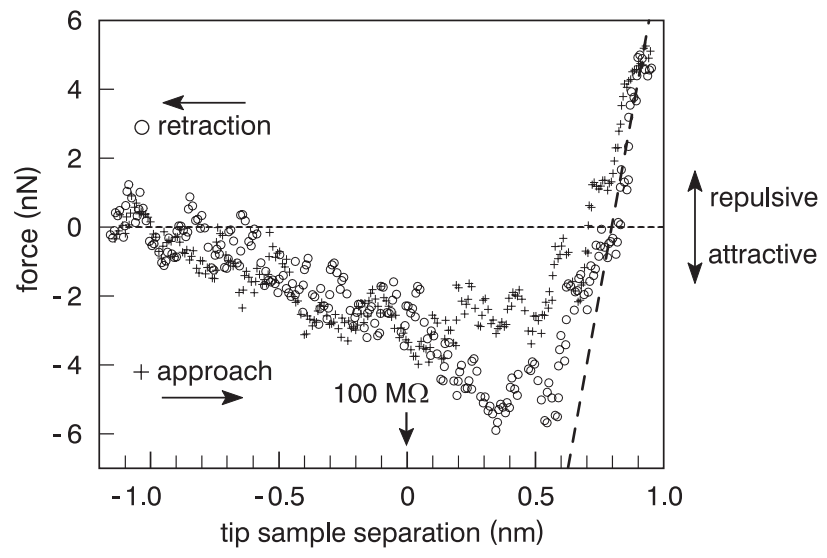

FIG. 3. Force versus tip-sample separation measured on a flat terrace using a W-trimer tip (tip-sample separation is defined as the relative motion of the tip with respect to the substrate using a tunnel resistance of $100 \mathrm{M} \Omega$ as the reference point). Note the hysteresis of $7 \mathrm{eV}$ between the approach and retraction curve, indicating that dissipative processes take place in the range of the adhesion maximum. Also note that no spontaneous jump to contact followed by the formation of an adhesion neck occurs. The attractive interaction has a length scale of $1 \mathrm{~nm}, 1$ order of magnitude larger than expected from universal scaling laws. The repulsive branch of the force curve is essentially linear (corresponding to a contact stiffness of $40 \pm 20 \mathrm{~N} / \mathrm{m}$, indicated by the dashed line) and reversible. Surprisingly, the tip-sample junction can support a repulsive load of at least $5 \mathrm{nN}$ corresponding to a contact pressure of $25 \mathrm{GPa}$. The compounded errors in determining the force scale correspond to $\pm 35 \%$; the compounded errors in the tip-sample separation $s$ are $\pm 20 \%$.

in metal contacts, and (4) the repulsive branch of the force curve is essentially linear and reversible.

The observation of repulsive forces implies that the tip atoms strongly interact with the Au surface atoms. However, no adhesion neck is formed between tip and substrate, which would manifest itself as a sudden increase of the attractive force due to surface tension [12,13]. Hence, we conclude that, in contrast to $\mathrm{Ni}$ [13] or $\mathrm{Au}$ [12] tips, spontaneous wetting of the W-trimer tip by $\mathrm{Au}$ substrate atoms does not occur. FIM images of the tip recorded after the approach experiments [see Fig. 2(b)] further support this conclusion. With the exception of two additional bright spots corresponding to adsorbed atoms, no structural changes of the tip are observed. In fact, the $\mathrm{W}$ trimer was recovered at the tip apex, which proves the outstanding mechanical stability of these atomic-sized W asperities.

The slope of the force curve in the repulsive regime (indicated by the dashed line) yields a contact stiffness of the order of $40 \pm 20 \mathrm{~N} / \mathrm{m}$ (the error is due to the systematic calibration errors associated with the force, $\pm 35 \%$, and the tip-sample separation, $\pm 20 \%$ ). Surprisingly, this number can be reproduced using standard elastic contact theory. The apex trimer presses against the Au surface, thereby inducing elastic deformations mainly of the latter (the elastic modulus of $\mathrm{W}$ is roughly 3.5 times that of $\mathrm{Au})$. The contact stiffness of a rigid circular punch in- denting a flat surface is $C_{P}=2 E r /\left(1-\nu^{2}\right)$ [14], where $E=125 \mathrm{GPa}$ and $\nu=0.38$ [15] are the elastic modulus and the Poisson number for $\mathrm{Au}$, respectively, and $r$ is the radius of the punch. Assuming that $r=0.25 \mathrm{~nm}$, which corresponds to the radius of a circle through the center of apex atoms, we obtain $C_{P} \approx 70 \mathrm{~N} / \mathrm{m}$. When comparing this number with the measured value, we must account for the finite stiffness of the repulsive interaction, which acts as an additional spring between tip and Au surface. As the binding energy of $\mathrm{Au}$ on $\mathrm{W}$ is comparable to that of bulk $\mathrm{Au}$, the interaction stiffness is estimated to be of the order of $C_{i} \simeq \pi r^{2} B / a \simeq 100 \mathrm{~N} / \mathrm{m}$, where $B=180 \mathrm{GPa}$ and $a=0.408 \mathrm{~nm}$ are the bulk modulus and the lattice constant of $\mathrm{Au}$, respectively. Hence, the measured stiffness is expected to be of the order of $1 /\left(C_{i}^{-1}+C_{P}^{-1}\right) \simeq 40 \mathrm{~N} / \mathrm{m}$, which is in good agreement with the experimental value. The experiment thus shows that standard continuum mechanics provides a quantitative framework to describe elastic deformations even on an atomic scale, a fact previously discovered in molecular dynamics simulations [13]. The plastic properties, on the other hand, are dramatically different from the macroscopic ones. The contact pressure reaches values of the order of $25 \mathrm{GPa}$ (maximum loading force divided by $\pi r^{2}$ ), which is more than 2 orders of magnitude larger than the macroscopic yield strength of Au. Material hardening in nanometer-scale structures is a general phenomenon $[12,13,16]$ that is intimately connected with the suppression of dislocation activity.

According to a theory by Rose et al. [17], the interaction between two metallic bodies should follow a universal law involving only two scaling parameters, namely the energy of adhesion and a length scale closely related to the screening length. Early experiments using Ir tips and Ir samples basically confirmed this theory [5]. However, the force curve measured in this experiment is clearly incompatible with the universal adhesion theory, which predicts that attractive forces should decrease exponentially with a decay length of the order of $0.1 \mathrm{~nm}$ [17]. The values we observe, $\approx 1 \mathrm{~nm}$, are one order of magnitude greater. In addition, we find a rather broad adhesion maximum, which also features a hysteresis of $7 \mathrm{eV}$ between approaching and retracting the tip from the sample. Hence we must conclude that important dissipative processes occur in this regime, which, however, have no net effect on the shape of the tip. A plausible mechanism must involve the displacement of atoms (most likely from the substrate) far away from their equilibrium position. From the small amount of energy that is dissipated, we conclude that only a few atoms in the immediate contact zone take part in this process.

Significant deviations from the universal behavior have been observed previously for $\mathrm{Au}$ and $\mathrm{Al}$ samples [6]. Interpretation of the data was ambiguous because of the uncertainty with respect to the tip structure. This issue has been resolved for the first time in this experiment. The results unambiguously show that one cannot extrapolate 
from bulk adhesion down to the atomic scale. It is known from theoretical investigations [18] that the electronic structure at the apex of a W tip deviates substantially from that of a bulk surface, which in turn may influence the adhesion characteristics. However, the effect is expected to be rather small as the adhesion interaction is sensitive to the integrated density of states. Correspondingly, electronic band-structure anomalies are averaged out [18].

We can also rule out Van der Waals interactions as an alternative explanation for the force curve anomalies. From the FIM images we deduce a tip radius of the order of $2.5 \mathrm{~nm}$. Assuming a typical value of $3 \times 10^{-19} \mathrm{~J}$ for the Hamaker constant we obtain adhesion forces that are at least 1 order of magnitude smaller than the measured values. It is equally difficult to reconcile the broad adhesion maximum within this framework.

In our previous discussion we have implicitly assumed that the tip-sample separation $s$, as defined in our experiment, is a faithful measure of the actual width of the gap between the tip apex and the sample surface and, hence, that the measured force curve represents the gradient of the adhesion potential. This premise is justified only if relaxations are negligible and both the tip and the sample can be considered rigid bodies. However, analogous to the discussion of repulsive interaction, we expect that the substrate also responds elastically to adhesion forces. Assuming that most of the adhesion force is due to the trimer-substrate interaction, the elastic sample stiffness is again of the order of $70 \mathrm{~N} / \mathrm{m}$. Correspondingly, the maximum elastic substrate response is of the order of $0.1 \mathrm{~nm}$. This value is clearly too small to explain the width of the adhesion maxima in the approach and the retraction curves, but it accounts for a substantial part of the tipsample motion in the positive force-gradient regime of the force curve close to contact. Hence, we conclude that the interaction potential actually varies more rapidly in this regime than the force curve suggests. The broad adhesion maxima and the shallow rolloff of the adhesion curve, on the other hand, cannot be reconciled within this simple response picture.

In summary, we have measured for the first time the force of interaction between an atomically characterized $\mathrm{W}$ trimer tip and a $\mathrm{Au}(111)$ surface. We find attractive adhesion forces of the order of several nanonewtons over a rather large range of tip-sample distance, which is incompatible with the standard theory of metal adhesion. We also find that the tip-sample system can support a contact pressure of up to $25 \mathrm{GPa}$ with no observable changes in the atomic structure of the tip. Finally, the measured contact stiffness of $40 \mathrm{~N} / \mathrm{m}$ can be explained within the framework of standard elastic theory.

The authors thank S. Jarvis for stimulating discussions. $\mathrm{Au}$ samples were kindly provided by E. Delamarche. The work at McGill University is supported by the National Science and Engineering Research Council (NSERC) of Canada and Le Fonds pour la Formation des Chercheurs et l'Aide à la Recherche (FCAR) de la Province de Québec.

*Corresponding author.

[1] A. R. H. Clarke, J. B. Pethica, J. A. Nieminen, F. Besenbacher, E. Lægsgaard, and I. Stensgaard, Phys. Rev. Lett. 76, 1276 (1996).

[2] S. Ciraci, E. Tekman, A. Baratoff, and I. P. Batra, Phys. Rev. B 46, 10411 (1992).

[3] D. M. Eigler and E. K. Schweizer, Nature (London) 344, 524 (1990).

[4] T. A. Jung, R. R. Schlittler, J. K. Gimzewski, H. Tang, and C. Joachim, Science 271, 181 (1996).

[5] U. Dürig, O. Züger, and D. W. Pohl, Phys. Rev. Lett. 65, 346 (1990).

[6] U. Dürig, O. Züger, L.C. Wang, and H. J. Kreuzer, Europhys. Lett. 23, 147 (1993).

[7] S. P. Jarvis, H. Yamada, S.-I. Yamamoto, H. Tokumoto, and J. B. Pethica, Nature (London) 384, 247 (1996).

[8] N. Sasaki and M. Tsukuda, Phys. Rev. B 52, 8471 (1995).

[9] For details, see A. Stalder, Ph.D. thesis, University of Fribourg, Switzerland, 1995.

[10] A. Stalder and U. Dürig, Rev. Sci. Instrum. 64, 3644 (1993).

[11] H.-W. Fink, IBM J. Res. Dev. 30, 460 (1986).

[12] A. Stalder and U. Dürig, Appl. Phys. Lett. 68, 637 (1996).

[13] U. Landman, W. D. Luedtke, and E. Ringer, Wear 153, 3 (1992).

[14] K. L. Johnson, Contact Mechanics (Cambridge University Press, Cambridge, England, 1985), Chap. 3.

[15] The elastic constants are calculated using the constitutive relations $E=9 G B /(G+3 B)$ and $\nu=1 / 2-$ $3 B / 2(G+3 B)$, where $B=180 \mathrm{GPa}$ and $G=45 \mathrm{GPa}$ are the bulk and the shear modulus, respectively [for numerical values, see, e.g., Ch. Kittel, Introduction to Solid State Physics (Wiley, New York, 1967), p. 122, 3rd ed.]

[16] G. Rubio, N. Agraït, and S. Vieira, Phys. Rev. Lett. 76, 2302 (1996).

[17] J. H. Rose, J. R. Smith, and J. Ferrante, Phys. Rev. B 28, 1835 (1983).

[18] H. Ness and F. Gauthier, J. Phys. Condens. Matter 7, 6625 (1995); 7, 6641 (1995). 